Robust Architecctures For Complex Multi-Agent Heterogeneous Systems

Naira Hovakimyan

UNIVERSITY OF ILLINOIS

$07 / 23 / 2014$

Final Report

DISTRIBUTION A: Distribution approved for public release.

Air Force Research Laboratory

AF Office Of Scientific Research (AFOSR)/ RTA

Arlington, Virginia 22203

Air Force Materiel Command 


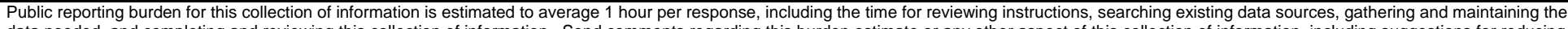

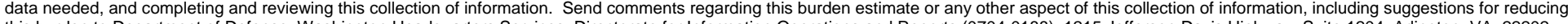

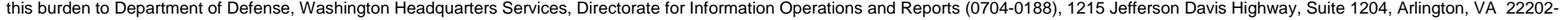

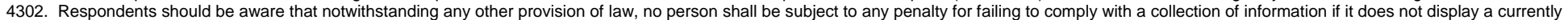
valid OMB control number. PLEASE DO NOT RETURN YOUR FORM TO THE ABOVE ADDRESS.

\begin{tabular}{|l|l|l|}
\hline 1. REPORT DATE (DD-MM- $Y Y Y Y)$ & 2. REPORT TYPE & 3. DATES COVERED (FrOm - To)
\end{tabular}

07/10/2014

final

$6 / 15 / 2011-6 / 14 / 2014$

4. TITLE AND SUBTITLE

Robust Architectures for Complex Multi-Agent Systems

5a. CONTRACT NUMBER

FA9550-11-1-0145

5b. GRANT NUMBER

5c. PROGRAM ELEMENT NUMBER

6. AUTHOR(S)

Naira Hovakimyan

5d. PROJECT NUMBER

5e. TASK NUMBER

5f. WORK UNIT NUMBER

8. PERFORMING ORGANIZATION REPORT NUMBER

University of Illinois at Urbana-Champaign, 1206 West Green street, Urbana, IL 61801

A1116 2010-07463

9. SPONSORING / MONITORING AGENCY NAME(S) AND ADDRESS(ES)

10. SPONSOR/MONITOR'S ACRONYM(S)

AFOSR

11. SPONSOR/MONITOR'S REPORT NUMBER(S)

12. DISTRIBUTION / AVAILABILITY STATEMENT

public

13. SUPPLEMENTARY NOTES

14. ABSTRACT

The research accomplished within this time period leveraged the prior accomplishments in the area of networked multi-agent systems. The past work (prior to 2011) focused on development of cooperative control algorithms in the presence of temporal and spatial constraints. During 2011-2014, we enriched the framework by i) developing cooperative control algorithms in the presence of dynamic information flow and quantized measurements [1], ii) developing a cooperative trajectory generation framework with account of spatial and temporal constraints [2], and iii) developing a decoupled architecture for distributed control of uncertain networked systems [3]. Additionally, a preliminary collision avoidance algorithm has been developed for a team of cooperating Unmanned Aerial Vehicles (UAV), executing a time-critical mission while encountering a non-cooperative aircraft.

\section{SUBJECT TERMS}

control of complex networked systems, performance guarantees

\begin{tabular}{|c|c|c|c|c|c|}
\hline \multicolumn{3}{|c|}{ 16. SECURITY CLASSIFICATION OF: } & \multirow{2}{*}{$\begin{array}{l}\text { 17. LIMITATION } \\
\text { OF ABSTRACT }\end{array}$} & \multirow{2}{*}{$\begin{array}{l}\text { 18. NUMBER } \\
\text { OF PAGES }\end{array}$} & \multirow{2}{*}{$\begin{array}{l}\text { 19a. NAME OF RESPONSIBLE PERSON } \\
\text { 19b. TELEPHONE NUMBER (include area } \\
\text { code) }\end{array}$} \\
\hline a. REPORT & b. ABSTRACT & c. THIS PAGE & & & \\
\hline
\end{tabular}




\title{
Robust Architectures for Complex Multi-Agent Systems
}

FA9550-11-1-0145

\section{FINAL REPORT}

Naira Hovakimyan

Mechanical Science and Engineering

University of Illinois at Urbana-Champaign, IL

\begin{abstract}
The research accomplished within this time period leveraged the prior accomplishments in the area of networked multi-agent systems. The past work (prior to 2011) focused on development of cooperative control algorithms in the presence of temporal and spatial constraints. During 2011-2014, we enriched the framework by i) developing cooperative control algorithms in the presence of dynamic information flow and quantized measurements [1], ii) developing a cooperative trajectory generation framework with account of spatial and temporal constraints [2], and iii) developing $a$ decoupled architecture for distributed control of uncertain networked systems [3]. Additionally, a preliminary collision avoidance algorithm has been developed for a team of cooperating Unmanned Aerial Vehicles (UAV), executing a time-critical mission while encountering a non-cooperative aircraft.
\end{abstract}

Main accomplishments: The conceptual architecture of the cooperative control framework is depicted in

Figure 1. The block diagram shows the interaction between the essential components of the architecture, such as the Distributed Trajectory Generation, Collision Avoidance, Time Coordination, and Path Following algorithms. The vehicles are exchanging information over a supporting communications network.

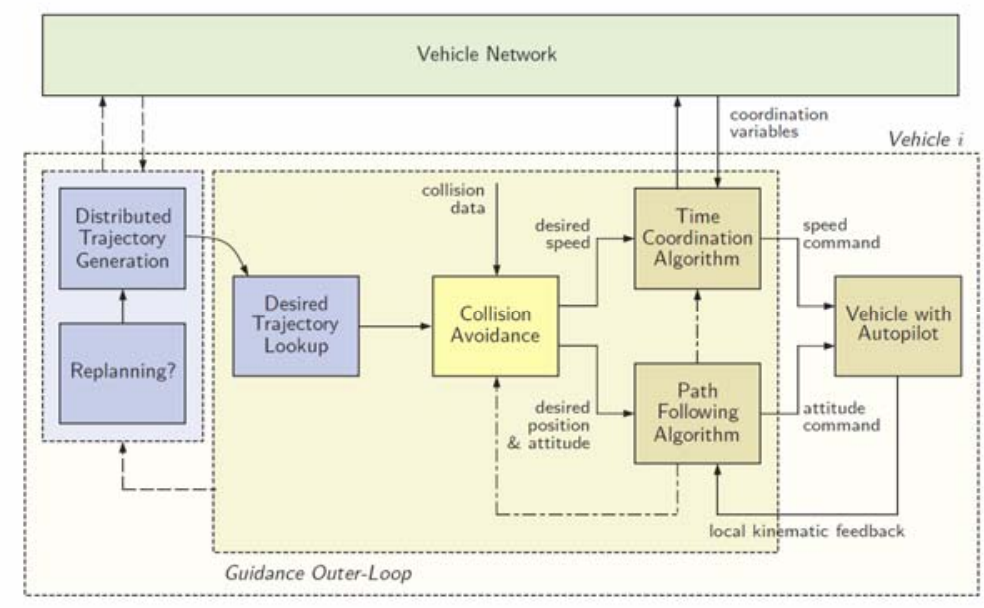


Figure 1 Cooperative Control Framework.

Several advances in these key components have been made over the reporting period. In particular, the convergence properties of a proportional-integral protocol for coordination of a network of agents with dynamic information flow and quantized measurements have been derived, when each agent is only required to exchange its coordination state with its neighboring agents, and the desired reference rate is only available to a group of leaders, [1]. The convergence of the collective dynamics is shown, when the graph that captures the network topology is not connected during some interval of time or even fails to be connected at all times. Lower bounds on the convergence rate of the collective dynamics as a function of the number of leaders and the Quality of Service (QoS) of the network are derived, which represent a measure of the level of connectivity of the dynamic graph that captures the underlying network topology. The convergence properties of the collective dynamics under quantized feedback are analyzed, where the solutions are interpreted in Krasovskii sense. We showed that in a fully quantized system one can afford much coarser quantization rate than in a partially quantized system.

In terms of algorithms for cooperative trajectory generation, Bézier curves have been explored and found useful for accommodating spatial deconfliction constraints. The Gilbert-Johnson-Keerthi algorithm (for computation of the minimum distance between convex shapes) along with de Casteljau algorithm (for subdividing the curve) have been explored for iteratively converging to the points on two Bézier curves that define the minimum distance in between them, [2]. Rational Bézier curves with tuning weights have been explored to accommodate more mission-specific constraints, and new subdivision points have been proposed that show faster convergence rates in determining the minimum distance between curves involving rational Bézier curves. Preliminary results show that using Bezier curves for the generation of feasible trajectories for a team of cooperating UAVs, result in more computationally efficient algorithms that may lend themselves for (near) real-time generation of these trajectories onboard the vehicles in the near future.

Design of collision avoidance algorithms, partly in support of the national effort in the safe integration of small UAVs in the National Airspace Systems, is also one of the main efforts within the development of this cooperative control framework. Results are obtained for a team of cooperating UAVs, avoiding a non-cooperating vehicle that is obstructing the area of operation and hampering the progress of the mission. It is shown that through speed adjustments of the collective team of UAVs, the non-cooperating vehicle is successfully avoided while ensuring coordination at all times. Hence, intervehicle collisions are prevented and the UAVs are able to successfully execute the mission and meet the critical time constraints. The results of this part of the research are reported in [4].

We also developed a control architecture that enables decoupling of the design of a robust controller with the communication protocol to the maximum extent, even in the presence of physical interconnections, [3]. With this architecture, one can integrate the existing communication protocols and robust control techniques into a unified system without worrying about the coupling between the control loop and the communication protocol. 
Although all communication models can be integrated into this framework, we choose decentralized event-triggering model as an example to demonstrate the performance of the proposed architecture. Stability conditions have been derived for the resulting closedloop systems in terms of communication and control parameters. Moreover, we showed that the transient performance of the communication-limited, uncertain system can be rendered arbitrarily close to a reference model by tuning the bandwidth of the low-pass filter in the local robust controller. The reference model in turn can be arbitrarily close to an ideal mathematical model, given sufficient communication resources.

Other theoretical results that may find their application in the overall cooperative control framework, are the developments of a real-time implementation of output-feedback $L_{1}$ adaptive controller over real-time networks [5], and an $L_{1}$ Simplex enabling fault-tolerant control of Cyber-Physical Systems [6]. In the development of an output-feedback $L_{1}$ adaptive controller over real-time networks, the system under consideration is networked with transmission delays, and it contains unmatched nonlinear uncertainties. We use event-triggering to trigger data transmissions from the plant (controller) to the controller (plant). An output-feedback strategy is provided based on the $L_{1}$ adaptive control architecture [7]. Stability conditions were derived, which establish the tradeoff between the control performance and the QoS of the communications network. We also derived the performance bound on the difference between the states in the uncertain networked control system and a stable ideal model, in terms of the event thresholds, the allowable transmission delays, and the adaptation rate. This bound can be further reduced by improving the QoS, which means that the performance of the uncertain system is subject to the hardware limitations.

The development of the $L_{1}$ Simplex addresses the concerns regarding system failures that comprise of physical failures as well as software failures. As the complexity of CyberPhysical Systems (CPS) increases, it becomes more and more challenging to ensure the reliability of CPS, especially in the presence of system failures. Simplex architecture is shown to be an efficient tool to address the software failure in such systems. However, when physical failures also appear, Simplex does not work anymore because the physical dynamics change due to physical failures. The Simplex architecture designed for the original physical model may not be suitable for the new dynamics. To address both software and physical failures, the L1Simplex architecture was developed, which contains the safety monitor, the high-performance controller (HPC), the $L_{1}$-based highassurance controller (HAC), and the decision logic for controller switching. The safety monitor is used to monitor the system behavior. It leads to another controller switching rule besides the stability-envelope-based rule in the decision logic. The HAC is designed based on the $L_{1}$ adaptive controller. With this HAC, the stability envelope is computed, which can be further extended by using multiple controllers in the HAC module. We showed that the $L_{1}$ Simplex architecture can efficiently handle a class of software and physical failures.

We leveraged NASA funding in the area of integration of UAVs into National Airspace Systems, which provides appropriate opportunity to transition the basic research results obtained within our AFOSR grant. 


\section{Acknowledgment / Disclaimer}

This work was sponsored (in part) by the Air Force Office of Scientific Research, USAF, under grant FA9550-09-1-0265. The views and conclusions contained herein are those of the author and should not be interpreted as necessarily representing the official policies or endorsements, either expressed or implied, of the AFOSR or the U.S. Government.

\section{References}

1. E. Xargay, R. Choe, N. Hovakimyan, I. Kaminer, "Multi-Leader Coordination Algorithm for Networks with Switching Topology and Quantized Measurements," Automatica, Vol. 50, No. 3, March 2014, pp. 841-851.

2. R. Choe, V. Cichella, E. Xargay, N. Hovakimyan, A.C. Trujillo, I. Kaminer, "A Trajectory-Generation Framework for Time-Critical Cooperative Missions," in Proceedings of AIAA InfoTech@Aerospace Conference, Boston, MA, August 2013, AIAA-2013-4582.

3. X. Wang, N. Hovakimyan, "Distributed Control of Uncertain Networked Systems: A Decoupled Design,” IEEE Transactions on Automatic Control, Vol. 58, No. 10, October 2013, pp. 2536-2549.

4. V. Cichella, R. Choe, S. B. Mehdi, E. Xargay, N. Hovakimyan, V. Dobrokhodov, I. Kaminer, "Trajectory Generation and Collision Avoidance for Safe Operation of Cooperating UAVs," in Proceedings of AIAA Guidance, Navigation and Control Conference, National Harbor, MD, January 2014, AIAA-2014-0972.

5. X. Wang, N. Hovakimyan, "Real_Time $L_{1}$ Adaptive Control for Uncertain Networked Control Systems,” IEEE Transactions on Automatic Control, 2014, accepted.

6. X. Wang, N. Hovakimyan, L. Sha, “ $L_{1}$ Simplex: Fault-Tolerant Control of CyberPhysical Systems,” X. Wang, N. Hovakimyan, L. Sha, L1Simplex: Fault-Tolerant Control of Cyber-Physical Systems, In Proceedings of ACM/IEEE $4^{\text {th }}$ International Conference on Cyber-Physical Systems, Philadelphia, PA, pp. 41-50, 2013 (one of the four finalists for "best paper award").

7. N. Hovakimyan, C. Cao, “ $L_{1}$ Adaptive Control Theory,” SIAM, 2010.

\section{Personnel supported partially by this grant}

1. Dapeng Li, Ph.D. Student (graduated in 2011)

2. Venanzio Cichella, Ph.D. Student

3. Ronald Choe, Ph.D. Student

4. Xiaofeng Wang, Postdoctoral Fellow (currently with Univ. of South Carolina)

5. Enric Xargay, Postdoctoral Fellow

6. Naira Hovakimyan, Professor and Schaller faculty scholar, Department of Mechanical Science and Engineering, University of Illinois at Urbana-Champaign, IL

\section{Selected publications (2011-2014) complementing above references:}

In Edited Volumes: 
1. I. Kaminer, E. Xargay, V. Cichella, N. Hovakimyan, A. Pascoal, P. Aguiar, V. Dobrokhodov, R. Ghabcheloo, Time-Critical Cooperative Path Following of Multiple UAVs: Case Studies, In Advances in Estimation, Navigation, and Spacecraft Control - Itzhack Y. Bar-Itzhack Memorial Symposium (Eds.: D. Choukroun, Y. Oshman, J. Thienel, and M. Idan), SpringerVerlag, 2014.

2. V. Kulkarni, H. Jiang, E. Kharisov, N. Hovakimyan, M. Riedel, K. Parhi, Synchronous Sequential Computations with Biomolecular Reactions. In V. Singh and P. Dhar (Ed.): Systems and Synthetic Biology Book, Springer Verlag, Netherlands, January 2014.

3. E. Xargay, N. Hovakimyan, V. Dobrokhodov, I. Kaminer, C. Cao, I. Gregory, $L_{1}$ Adaptive Control in Flight. In Intelligent Systems. Series: AIAA Progress in Aeronautics and Astronautics, pp. 129-172, 2012.

4. X. Wang, N. Hovakimyan, Performance Prediction in Uncertain Multi-Agent Systems Using $L_{1}$ Adaptation-Based Distributed Event-Triggering. In Distributed Decision Making and Control. Series: Lecture Notes in Control and Information Sciences, Springer Verlag, pp. 171-193, 2011.

\section{In Journals:}

5. K.-K. Kim, N. Hovakimyan, Multi-Criteria Optimization for Filter Design of $L_{1}$ Adaptive Control, Journal of Optimization Theory and Applications, 2013.

6. V. Kulkarni, E. Kharisov, N. Hovakimyan, J. Kim, Load Capacity Improvements in Nucleic Acid Based Systems Using Partially Open Feedback Control, ACS Synthetic Biology (IWBDA 2014 Special Issue).

7. E. Kharisov, N. Hovakimyan, K. Astrom, Comparison of Architectures and Robustness of MRAC and $L_{1}$ Adaptive Controllers, International Journal of Adaptive Control and Signal Processing, 2013.

8. E. Xargay, I. Kaminer, A. Pascoal, N. Hovakimyan, V. Dobrokhodov, V. Cichella, A. Aguiar, R. Ghabcheloo, Time-Critical Cooperative Path Following of Multiple UAVs over TimeVarying Networks, AIAA Journal of Guidance, Control and Dynamics, 2013.

9. D. Li, N. Hovakimyan, Positive Invariant Set for $L_{1}$ Adaptive Controller in the Presence of Input Saturation, International Journal of Adaptive Control and Signal Processing, vol. 27, No. 11, pp. 1012-1030, 2013.

10. X. Wang, N. Hovakimyan, Distributed Control of Uncertain Networked Control Systems: A Decoupled Design, IEEE Transactions on Automatic Control, vol. 58, No.10, pp. 2536-2549, 2013.

11. D. Li, N. Hovakimyan, Bode-like Integral for Continuous-Time Closed-Loop Systems in the Presence of Limited Information, IEEE Transactions on Automatic Control, vol. 58, No.6, pp. 1457-1469, 2013.

12. V. Dobrokhodov, E. Xargay N. Hovakimyan, I. Kaminer, C. Cao, I. Gregory, Multicriteria Analysis of an $L_{1}$ Adaptive Flight Control System, Proceedings of the Institution of Mechanical Engineers, Part I, Journal of Systems and Control Engineering, vol. 227, No. 4, pp.413-427, 2013.

13. L. Ma, N. Hovakimyan, Vision-Based Cyclic Pursuit for Cooperative Target Tracking, AIAA Journal of Guidance, Control and Dynamics, vol. 35, No.2, pp. 617-622, 2013.

14. D. Li, N. Hovakimyan, Bode-Like Integral for Stochastic Switched Systems in the Presence of Limited Information, Automatica, vol. 49, No.1, pp. 1-8, 2013. 
15. E. Xargay, V. Dobrokhodov, I. Kaminer, A. Pascoal, N. Hovakimyan, C. Cao, Time-Critical Cooperative Control of Multiple Autonomous Vehicles, IEEE Control Systems Magazine, pp. 49-73, October, 2012.

16. X. Wang, Y. Sun, N. Hovakimyan, Asynchronous Task Execution in Decentralized EventTriggered Networked Control Systems, Systems and Control Letters, vol. 61, No. 9, pp. 936944, 2012.

17. X. Wang, N. Hovakimyan, $L_{1}$ Adaptive Controller for Nonlinear Time-Varying Reference Systems, Systems and Control Letters, vol. 61, No. 4, pp. 455-463, 2012.

18. H. Sun, N. Hovakimyan, T. Basar, $L_{1}$ Adaptive Controller for Uncertain Nonlinear MultiInput Multi-Output Systems with Input Quantization, IEEE Transactions on Automatic Control, vol. 57, No. 1, pp. 565-578, 2012.

19. N. Hovakimyan, C. Cao, E. Kharisov, E. Xargay, I. Gregory, $L_{1}$ Adaptive Controller for Safety Critical Systems: Guaranteed Robustness with Fast Adaptation, IEEE Control Systems Magazine, pp. 54-104, October, 2011.

20. V. Dobrokhodov, I. Kaminer, I. Kitsios, E. Xargay, N. Hovakimyan, C. Cao, I. Gregory, L. Valavani, Experimental Validation of $L_{1}$ Adaptive Control: Rohrs' Counterexample in Flight, AIAA Journal of Guidance, Control and Dynamics, vol. 34, No. 5, pp.1311-1328, 2011.

\section{In Conferences:}

1. S. Bhattacharya, T. Basar, N. Hovakimyan, On the Construction of Barrier in Visibility based Pursuit-Evasion Game, European Control Conference, Strasbourg, France, 2014.

2. K. Ackerman, S. Pelech, R. Carbonari, N. Hovakimyan, A. Kirlik, Pilot-in-the-Loop Flight Simulator for NASA's Transport Class Model, In Proceedings of AIAA Guidance, Navigation, and Control Conference, Washington DC, AIAA 2014-0613, 2014.

3. H. Lee, S. Snyder, N. Hovakimyan, An Adaptive Unknown Input Observer for Fault Detection and Isolation of Aircraft Actuator Faults, In Proceedings of AIAA Guidance, Navigation, and Control Conference, Washington DC, AIAA 2014-0266, 2014.

4. H. Sun, N. Hovakimyan, T. Basar, Reference Tracking of Uncertain Nonlinear Multi-Input Multi-Output Quantized Systems using $L_{1}$ Adaptive Control, In Proceedings of $52^{\text {nd }}$ IEEE Conference on Decision and Control, Florence, Italy, pp. 7546-7551, 2013.

5. H. Sun, Z. Li, N. Hovakimyan, T. Basar, Performance Prediction of $L_{1}$ Adaptive Control in Linear Stochastic Systems, In Proceedings of $52^{\text {nd }}$ IEEE Conference on Decision and Control, Florence, Italy, pp. 606-611, 2013.

6. V. Cichella, R. Choe, S. B. Mehdi, E. Xargay. N. Hovakimyan, I. Kaminer, and V. Dobrokhodov, A 3D Path-Following Approach for a Multirotor UAV on SO(3), In Proceedings of 2nd IFAC RED-UAS 2013 Workshop on Research, Education and Development of Unmanned Aerial Systems, Compiegne, France, 2013.

7. K.-D. Nguyen, H. Dankowicz, N. Hovakimyan, Marginal Stability in $L_{1}$ Adaptive Control of Manipulators, In Proceedings of the ASME 2013 International Design Engineering Technical Conferences \& Computers and Information in Engineering Conference, IDETC/CIE 2013, Portland, OR, DETC2013-12744, 2013 (one of the five finalists for "best student paper award").

8. R. Choe, V. Cichella, E. Xargay, N. Hovakimyan, I. Kaminer, Cooperative Path Following and Collision Avoidance along Feasible Trajectories for UAVs, In Proceedings of AIAA Guidance, Navigation and Control Conference, Boston, MA, AIAA-2013-4582, 2013. 
9. V. Cichella, I. Kaminer, V. Dobrokhodov, N. Hovakimyan, Cooperative Vision-based Tracking of Multiple UAVs, In Proceedings of AIAA Guidance, Navigation and Control Conference, Boston, MA, AIAA-2013-5110, 2013.

10. R. Choe, E. Xargay, and N. Hovakimyan, $L_{1}$ Adaptive Control for a Class of Nonaffine-inControl Nonlinear Systems, In Proceedings of IFAC International Workshop on Adaptation and Learning in Control and Signal Processing, Caen, France, July 2013.

11. X. Wang, E. Kharisov, N. Hovakimyan, $L_{1}$ Adaptive Control of Uncertain Networked Control Systems, In Proceedings of American Control Conference, Washington, DC, pp. 4122-4127, 2013.

12. J. Vanness, E. Kharisov, N. Hovakimyan, $L_{1}$ Adaptive Output-Feedback Controller for Linear Time-Varying Reference Systems, In Proceedings of American Control Conference, Washington, DC, pp. 4183-4188, 2013.

13. L. Ma, N. Hovakimyan, Cooperative Target Tracking in Balanced Vehicular Formations: Multiple UAVs Tracking a Ground Vehicle, In Proceedings of American Control Conference, Washington, DC, pp. 5386-5391, 2013.

14. I. Kaminer, E. Xargay, V. Cichella, N. Hovakimyan, A. Pascoal,V. Dobrokhodov, TimeCritical Cooperative Path Following of Multiple UAVs: Case Studies, In Proceedings of Itzhack Y. Bar-Itzhack Memorial Symposium on Estimation, Navigation and Spacecraft Control, Technion, Haifa, Israel, 2012.

15. S. Bhattacharya, T. Basar, N. Hovakimyan, Game-theoretic analysis of a visibility based pursuit-evasion game in the presence of a circular obstacle, In Proceedings (1479) of American Institute of Physics, pp. 1222-1225, 2012.

16. V. Cichella, I. Kaminer, E. Xargay, V. Dobrokhodov, N. Hovakimyan, P. Aguiar, A. Pascoal, A Lyapunov-Based Approach for Time-Coordinated 3D Path-Following of Multiple Quadrotors in $\mathrm{SO}(3)$, In Proceedings of $51^{\text {st }}$ IEEE Conference on Decision and Control, Maui, HI, pp. 1776-1781, 2012.

17. J. Vanness, E. Kharisov, N. Hovakimyan, Generalization of Proportional Adaptation Law for $L_{1}$ Adaptive Controller, In Proceedings of $51^{\text {st }}$ IEEE Conference on Decision and Control, Maui, HI, pp. 3215-3220, 2012.

18. E. Xargay, R. Choe, N. Hovakimyan, I. Kaminer, Convergence of a PI Coordination Protocol in Networks with Switching Topology and Quantized Measurements, pp. 6107-6112, In Proceedings of 51 ${ }^{\text {st }}$ IEEE Conference on Decision and Control, Maui, HI, 2012.

19. Z. Li, H. Sun, N. Hovakimyan, $L_{1}$ Adaptive Controller for MIMO System with Unmatched Uncertainties Using Modified Piecewise Constant Adaptation Law, In Proceedings of $51^{\text {st }}$ IEEE Conference on Decision and Control, Maui, HI, pp. 7303-7308, 2012.

20. Z. Li, N. Hovakimyan, G.-O. Kaasa, Bottomhole Pressure Estimation and $L_{1}$ Adaptive Control in Managed Pressure Drilling System, In Proceedings of $1^{\text {st }}$ IFAC Workshop on Automatic Control of Offshore Oil and Gas Production, Trondheim, Norway, pp. 128-133, 2012.

21. H. Sun, Z. Li, N. Hovakimyan, T. Basar, G. Downton, $L_{1}$ Adaptive Control for Directional Drilling Systems, In Proceedings of $1^{\text {st }}$ IFAC Workshop on Automatic Control of Offshore Oil and Gas Production, Trondheim, Norway, pp. 72-77, 2012.

22. E. Kharisov, N. Hovakimyan, Generalization of $L_{1}$ Adaptive Control Architecture for Switching Estimation Laws, In Proceedings of American Control Conference, Montreal, Canada, pp. 1907 - 1912, 2012. 
23. J. Vanness, E. Kharisov, N. Hovakimyan, $L_{1}$ Adaptive Control with Proportional Adaptation Laws, In Proceedings of American Control Conference, Montreal, Canada, pp. 1919 - 1924, 2012.

24. M. Naghnaeian, P. Voulgaris, N. Hovakimyan, On the Robustness of $L_{1}$ Adaptive Control Law with Time-Varying Perturbations and Filter Design, In Proceedings of American Control Conference, Montreal, Canada, pp. 1937 - 1942, 2012.

25. X. Wang, N. Hovakimyan, A Decoupled Design in Distributed Control of Uncertain Networked Control Systems, In Proceedings of American Control Conference, Montreal, Canada, pp. 6497 - 6502, 2012.

26. K.-K. Kim, E. Kharisov, N. Hovakimyan, Filter Design for $L_{1}$ Adaptive Output Feedback Controller, In Proceedings of $50^{\text {th }}$ IEEE Conference on Decision and Control, Orlando, FL, pp. 5653 - 5658, 2011.

27. S. Bhattacharya, T. Basar, N. Hovakimyan, Non-cooperative Strategies for Connectivity Maintenance among Autonomous Mobile Agents, In Proceedings of $50^{\text {th }}$ IEEE Conference on Decision and Control, Orlando, pp. 261 - 266, FL, 2011.

28. D. Li, N. Hovakimyan, Optimal State estimation over Gaussian Channels via Noiseless Feedback, In Proceedings of $50^{\text {th }}$ IEEE Conference on Decision and Control, Orlando, FL, pp. 2608 - 2613, 2011.

29. Z. Li, N. Hovakimyan, G.-O. Kaasa, Fast Estimation and $L_{1}$ Adaptive Control for Bottomhole Pressure in Managed Pressure Drilling, In Proceedings of IEEE Multi-Conference on Systems and Control, pp. 996-1001, Denver, CO, 2011.

30. H. Sun, Z. Li, N. Hovakimyan, T. Basar, G. Downton, $L_{1}$ Adaptive Controller for a Rotary Steerable System, In Proceedings of IEEE Multi-Conference on Systems and Control, pp. 1020-1025, Denver, CO, 2011.

31. X. Wang, H. Sun, N. Hovakimyan, T. Basar, Bounds on Transmission Rates and Performance for Quantized Networked Systems, In Proceedings of $18^{\text {th }}$ IFAC World Congress, Milan, Italy, 2011.

32. H. Sun, N. Hovakimyan, T. Basar, $L_{1}$ Adaptive Controller for Uncertain Nonlinear MultiInput Multi-Output Systems with Input Quantization, In Proceedings of $18^{\text {th }}$ IFAC World Congress, Milan, Italy, 2011.

33. R. Choe, N. Hovakimyan, Perching Maneuver for an MAV Augmented with an $L_{1}$ Adaptive Controller, In Proceedings of AIAA Guidance, Navigation and Control Conference, Portland, OR, AIAA-2011-6455, 2011.

34. V. Cichella, E. Xargay, V. Dobrokhodov, I. Kaminer, A. Pascoal, N. Hovakimyan, Geometric 3D Path-Following Control for a Fixed-wing UAV on SO(3), In Proceedings of AIAA Guidance, Navigation and Control Conference, Portland, OR, AIAA-2011-6415, 2011.

35. Z. Li, J. Vanness, D. Caballero, N. Hovakimyan, D. Stipanovic, Distributed Target Tracking and Collision Avoidance using Multiple Nonholonomic Robots with Uncertain Dynamics, In Proceedings of AIAA Guidance, Navigation and Control Conference, Portland, OR, AIAA2011-6479, 2011.

36. E. Kharisov, K.-K. Kim, X. Wang, and N. Hovakimyan, Limiting Behavior of $L_{1}$ Adaptive Controllers, In Proceedings of AIAA Guidance, Navigation and Control Conference, Portland, OR, AIAA-2011-6441, 2011.

37. L. Ma, N. Hovakimyan, Vision-Based Cyclic Pursuit for Cooperative Target Tracking, In Proceedings of American Control Conference, San Francisco, CA, pp. 4616-4621, 2011.

38. X. Wang, N. Hovakimyan, $L_{1}$ Adaptive Controller for Nonlinear Reference Systems, In Proceedings of American Control Conference, San Francisco, CA, pp. 594-599, 2011. 
39. Z. Li, N. Hovakimyan, D. Stipanovic, Distributed Multi-Agent Tracking and Estimation with Uncertain Agent Dynamics, In Proceedings of American Control Conference, San Francisco, CA, pp. 2204-2209, 2011.

40. H. Sun, N. Hovakimyan, T. Basar, $L_{1}$ Adaptive Controller for Quantized Systems, In Proceedings of American Control Conference, San Francisco, CA, pp. 582-587, 2011.

41. H. Sun, Q. Zhu, N. Hovakimyan, T. Basar, $L_{1}$ Adaptive Controller for Positive LTI Systems, In Proceedings of American Control Conference, San Francisco, CA, pp. 13 - 18, 2011.

42. E. Xargay, C. Langbort, N. Hovakimyan, $L_{1}$ Adaptive Controller for Optical Soliton Propagation, In Proceedings of American Control Conference, San Francisco, CA, pp. 7-12, 2011.

43. E. Kharisov, N. Hovakimyan, $L_{1}$ Adaptive Output Feedback Controller for Minimum Phase Systems, In Proceedings of American Control Conference, San Francisco, CA, pp. 11821187, 2011.

44. D. Li, N. Hovakimyan, Bode-Like Integral for Stochastic Switched Systems with Applications in Networked Control Systems and Macroeconomics, In Proceedings of American Control Conference, San Francisco, CA, pp. 2843 - 2848, 2011.

\section{Honors}

2014

2014

2014

2013

2012

2012

2012

$2011-2014$

$2011-2012$

2011
TUM-IAS honorary Hans Fischer senior fellow

Humboldt prize, Alexander von Humboldt Foundation, Germany

Keynote speaker, International Conference on Unmanned Aircraft Systems, Orlando, FL

Plenary speaker, II Midwest Control and Game Workshop, University of Notre Dame, IN

Technical Achievement Award, World Congress, $9^{\text {th }}$ International Conference on Mathematical Problems in Engineering, Aerospace and Sciences, Vienna, Austria

Best Presentation Award, AIAA Guidance, Navigation and Control Conference

Keynote speaker, ICNPAA World Congress: Mathematical Problems in Engineering, Sciences and Aerospace, Vienna, Austria

\section{University Scholar, UIUC}

Member of Engineering Faculty Leadership Forum of UIUC

AIAA Mechanics and Control of Flight Award, with the citation "For ground breaking work in $L_{1}$ robust adaptive control of nonlinear uncertain systems, vision-based guidance, navigation and control, and cooperative path planning of UAVs" 


\section{Transitions}

- Raymarine has successfully implemented $L_{1}$ Adaptive Control on their Evolution Autopilot series for marine vessels.

- Unmanned Dynamics multirotor solutions (educopters acquired by US Universities, phocopters acquired by Naval Air Warfare Center Weapons Division)

- The theory has been implemented on NASA's AirSTAR GTM dynamically scaled jet powered piloted aircraft and flight tested for post-stall flight regimes. POC: I. Gregory, NASA LaRC, Hampton, VA 23681, Ph: 757-864-4075, E-mail: i.m.gregory@larc.nasa.gov.

- The theory has been used to augment an existing autopilot (Piccolo) for accurate path following in the problem of time-critical cooperation of UAVs with spatial constraints in the presence of time-varying communication network topology. POC: Isaac Kaminer, MAE, NPS, Monterey, CA 93943, Phone: 831-656-3459 (further transition to USSOCOM in TNT exercises).

\section{New Discoveries}

2014, Feb. 9 Patent (control No.13/023,965) “Adaptive Control for Uncertain Nonlinear Multi-Input Multi-output Systems” (with C. Cao and E. Xargay) 